\title{
GENERAL PRINCIPLES OF INFECTION CONTROL AND SAFETY INITIATIVES
}

\author{
By \\ MOSTAFA M. ELNAKIB ${ }^{1}$, NAEMA MAHMOUD MOHAMED ${ }^{1}$ \\ and TOSSON A. MORSY ${ }^{2}$
}

Military Medical Academy, Cairo $1291^{1}$, and Faculty of Medicine, Ain Shams

University, Cairo $11566^{2}$, Egypt

\begin{abstract}
Standard precautions are guidelines established to break the infection chain and reduce risk of pathogen transmission in hospitals. Standard precautions apply to blood, body fluids, secretions and excretions (except sweat), non-intact skin, and mucous membranes.
\end{abstract}

Key words: infection control, Safety initiatives, Principles, General review

\section{Introduction}

Infection control is a discipline that applies epidemiologic, scientific principles and statistical analysis towards prevention or reduction in rates of nosocomial infections. Effective infection control programs proved to reduce nosocomial infections rates but also being cost-effective. Infection control is a key component of the broader discipline of hospital epidemiology (Haley et al, 1985).

The recognition that infectious agents can be transmitted within hospitals to susceptible patients and health care workers began in the days of Semmelweis, who noted that puerperal fever was associated with the lack of hand-washing by clinicians performing autopsies. This discovery, in turn, led to the introduction of hand dips with chlorinated lime at Vienna General Hospital. Over the years, these ideas evolved to form current guidelines about hand-washing, although Semmelweis promoted hand cleansing and was opposed to hand-washing with soap and water (Hall, 2007).

\section{Review and Discussion}

Infection control programs became a requirement in the United States largely as mandates of the Joint Commission for Accreditation of Hospitals (JCAHO) and the leadership guidelines and definitions of the $\mathrm{Ce}$ nters for Disease Control and Prevention (CDC). In order to achieve the main goal of preventing or reducing the risk of hospitalacquired infections, a hospital epidemiology program should have the following oversight functions and responsibilities: Surveil- lance, either hospital-wide or targeted Education about prevention of infections (e.g., by hand disinfection), outbreak investigations, cleaning disinfection, sterilization of equipment, disposal of infectious waste hospital employee health specifically after ex0posure to either blood-borne or respiratory pathogens and review its antibiotic utilization relationship to the local antibiotic resistance patterns, also, prevention of infections due to percutaneous intravascular devices and development of the infection control policies and procedures and oversight on the use of new products that directly or indirectly relate to the risk of nosocomial infections (Edmond and Wenzel, 2005).

On the basis of data collected in the Study on the Efficacy of Nosocomial Infection Control (SENIC), CDC (1987) recommended that all hospitals employ at least one infection control professional (ICP, frequently a nurse) for every 250 occupied beds. But, since these recommendations were done over 30 years ago, the ideal ratio of ICPs to patients is at present unknown.

Hospital infection control departments usually derive authority and communicate with other administrative components of the hospitals via an infection control committee. This committee typically includes representtatives from medical and surgical services, nursing, microbiology, hospital administration, and employee health (Taylor, 1996).

In order to be successful, infection control programs must have explicit and adequate administrative support, resources and an or- 
ganizational commitment to a safety culture.

Four major infection control areas are: 1Standard precautions, including hand hygiene. 2- Isolation precautions cleaning, dis-infection, 3- Environmental cleaning, and sterilization. 4- Surveillance (Siegel et al, 2007).

1- Standard Precautions: Various forms of isolation have been used in an attempt to reduce the spread of nosocomial infections (Garner, 1996a). In 1996, the CDC and Hospital Infection Control Advisory Committee (HICPAC) issued a new system of isolation precautions. These guidelines were updated in 2007 .Standard precautions are recommended in the care of all hospitalized patients. The category of standard precautions combines the important features of body substance isolation policies and universal precautions, and, by doing so, aims to reduce the risk of transmission of infectious agents between patient and healthcare worker (Garner, 1996b). Standard precautions apply to blood, all body fluids, no intact skin, mucous membranes and secretions and excretions except sweat. They entail: hand-washing before and after every patient contact, the use of gloves, gowns and eye protection in situations in which exposure to body secretions or blood is possible, hand-washing after gloves are removed, safe disposal of sharp instruments and needles in impervious containers and the placement of soiled linens in impervious bags and bloody or contaminated materials such as feces or urine in sanitary toilets (Whitehouse et al, 1998).

CDC (2007) guidelines included several additional components: safe injection practices, use of a mask when prolonged procedures involving puncture of the spinal canal are performed (such as myelography, epidural anesthesia, and injection of chemotherapeutic agents) and respiratory hygiene/ cough etiquette, which applies to all patients and accompanying family or friends who have any sign of respiratory illness such as cough, congestion, rhinorrhea or increased volumes of respiratory secretions. Such individuals should cover their nose or mouth when coughing, promptly dispose used tissues and practice hand hygiene after contact with respiratory secretions. The use of a mask and spatial separation of such patients in waiting or patient care areas is also recommended (Pittet et al, 2006).

a- Hand hygiene: refers to either hand-washing with soap and water or use of alcoholbased gels or foams that do not require the use of water. It is the single most important measure to reduce transmission of microorganisms from one person to another or one site to another on the same patient.

The primary problem with hand hygiene is laxity of practice, not a paucity of good products. As described in the next section, this is particularly true with hand-washing with soap and water compared to alcoholbased products. Evidence for the efficacy of hand hygiene primarily comes from studies related to methicillin-resistant St. aureus and is presented separately (Boyce and Pittet, 2002).

b- Soap and water: CDC guidelines advise use of plain (no antimicrobial) soap with water for routine hand-washing, with use of an antimicrobial agent for specific circumstances. There are limited data with respect to the choice of plain soap, antiseptic soap, or antiseptic hand rub (Gould et al, 2007).

There are a number of problems with hand-washing with soap and water: Compliance with hand-washing rarely exceeds $45 \%$ even under study conditions, and even in intensive care units (Pittet et al, 2002). Even when hand-washing is performed, proper technique is usually not followed. In one report, for example, the mean observed washing time was less than 10 seconds in most cases, compared with 15 to 30 seconds (Eckmanns et al, 2006). The importance of duration of hand-washing was illustrated in a study in which vancomycin-resistant enterococci (VRE) was placed onto the hands of healthy volunteers. Thirteen second washes with water and soap was necessary to complete eradicate VRE hand carriage. But, a five second wash with water alone caused 
virtually no change in VRE recovery (Noskin et al, 1995). The frequency of handwashing by HCW is affected by accessibility of sinks and by characteristics of soap used (e.g., its smell, consistency, color, and even the ease where the soap induces lathering).

Besides, the location and type of soap dispensers can affect compliance. Hand-washing with plain soap does not consistently or reliably prevent microbial transmission. Frequent hand-washing causes skin damage, with resultant changes in microbial flora, increased skin shedding, and the risk of transmission of microorganisms.

c- Alcohol containing hand disinfection: Alcohol containing hand disinfection (AHD) is an effective and practical alternative to standard soap and water. Such alcohol-based products have rapid antimicrobial effects and are equally effective against gram-positive and gram-negative organisms (when compared with chlorhexidine). Alcohol-based preparations also require less time than chlorhexidine gluconate to affect a maximum reduction in bacteria counts and are at least as tolerable on skin as are antiseptic detergents.

Compared to hand-washing with soap and water, AHD is easier to perform and takes less time. This was illustrated in a study that estimated the time required for hand-washing and bedside AHD in a typical 14-bed intensive care unit (ICU) with 12 healthcare workers, using an ideal duration of handwashing of 40 to 80 seconds and 20 seconds for AHD. If $100 \%$ compliance were achieved, hand-washing would consume 16 hours of nursing time per day shift, com-pared with only three hours required for the AHD (Pittet et al, 2000). These benefits have led to improved compliance and reductions in nosocomial infections. In a report from a Swiss teaching hospital, introduction of a bedside alcohol-based hand disinfectant led to a significant improvement in hand hygiene compliance and decreased the nosocomial infection rates (9.9 vs. 16.9\%) and transmission of methicillin-resistant St. aureus, 0.9 vs. 2.2 episodes/10,000 patient days (Larson,
1999).

Although AHD improves hand hygiene adherence rates, it is only one component for prevention of infection. A crossover trial of alcohol based hand gel demonstrated that improved hand hygiene adherence was not associated with detectable changes in the incidence of healthcare-associated infection, although the study was underpowered to detect a statistically significant effect. An important limitation of AHD is that it does not reduce the incidence of Clostridium difficile colitis. This reflects the lack of activity of alcohol-based hand rubs against sporeforming bacteria (Gordin et al, 2005). Waterless alcohol-based hand rubs must be available at bedside and that their use should be promoted over hand-washing with soap and water. Because AHD is associated with a tendency to desiccate skin, the additional use of an appropriate emollient or barrier cream to further protect the skin is important. The AHD is more efficient than handwashing with soap and water. The detailed review of antimicrobial ingredients designed for hand-washing is presented in the CDC guidelines (Rupp et al, 2008). But, if a facility is experiencing a $C$. difficile out-break it is prudent for health care workers to wash their hands with soap and water rather than an alcohol-based hand sanitizer.

d- Fingernails: Much of the resident micro flora of hands is found in the periungual and subungual areas, and often fingernails are neglected during routine hand cleansing. When the fingernails are long and when artificial fingernails are worn, there is an increase in periungual colonization with a variety of pathogens (Pottinger et al, 1989).

Both the artificial fingernails and infected native fingernails have been epidemiologically linked to the infection outbreaks among the intensive care unit patients, neonates, and patients undergoing surgery (Moolenaar et al, 2000). Freshly applied nail polish does not increase the number of bacteria recovered from periungual skin, but chipped nail polish has been associated with increased 
numbers of organisms on fingernails (Passaro et al, 1997). These organisms are not routinely removed by careful hand-washing or even by surgical scrubs.

Guidelines from the CDC and Association of Operating Room Nurses (AORN) prohibit the use of artificial fingernails or extenders by health care workers with "direct contact with patients at high risk" e.g., those in the intensive care units or operating rooms. However, the policies in many United States hospitals that prohibit the use of artificial nails in all health care workers with direct patient contact. There is no consensus on the need to prohibit wearing of rings in healthcare settings even though several studies have shown that skin beneath rings is more heavily colonized with bacteria than adjacent skin not covered by rings (Rubin, 1988).

e- Gloves: There are three important reasons why gloves should be worn by hospital personnel: To provide a protective barrier for the hands from contaminated material such as blood or body fluids, or other potentially infectious material (such as vomit or feces) or from contaminated equipment, to reduce the acquisition of micro-organisms from a patient who is colonized or infected with pathogenic organisms, to reduce the microorganisms transmission from the hands of hospital staff to patients. However, wearing gloves does not replace the need for hand-washing because gloves may have small defects or tears that are unapparent, and because hands can become contaminated during removal of gloves (Alrawi et al, 2001). This was reported that examined 206 glove pairs after they were worn by surgeons. Unrecognized perforations were noted in $14 \%$; the majority was on thumb and index fingers of the gloves. Surgical experience, type of gloves, type of surgery, and double gloving did not affect rates and sites of perforation. Reinforcement of the thumb and index fingers of gloves may be a way to reduce perforation risk.

A study of washing gloved hands inoculated with selected nosocomial pathogens sho- wed that organisms (St. aureus) could still be recovered from hands after gloves removal. These findings are the principle reason why good hand-washing after glove removal is essential. Gloves must be chang- ed between patient contacts. It is sometimes important to change heavily contaminated gloves while caring for a single patient to prevent cross-contamination of body sites or if contamination of portable equipment can occur (Doebbeling et al, 1988).

f- Masks: Masks are used for three purposes in infection control: To protect healthcare personnel from infectious material from patients (such as respiratory secretions and aerosols containing blood or body fluids), to protect patients undergoing sterile procedures from respiratory aerosols from healthcare personnel and to limit spread of infectious respiratory secretions from coughing patients. Masks should not be confused with particulate respirators that are used to prevent transmission by airborne droplet nuclei of infectious agents as $M$. tuberculosis.

2- Isolation Precautions: In addition to the standard precautions, there are three isolation categories that reflect the major modes of microorganism transmission in the nosocomial settings: contact, droplet, and airborne spread. The rooms of patients' requiring contact precautions should be clearly marked with instructions regarding the type of precautions that must be observed. Ample supplies should be readily available outside the patient room to facilitate adherence, and hospital policies should be enforced (Muto et al, 2003).

a- Contact Precautions: Microorganisms can be transmitted to a patient by contact between the patient and a healthcare worker, or by contact between the patient and a contaminated object; such as toys, clothing, inadequately disinfected endoscopes or other medical equipment (Rey, 1999). Contact precautions should be used in the care of patients with multidrug-resistant bacteria, and various enteric, parasitic, and viral pathogens. Ideally, patients who require contact 
precautions should either be in a private room or cohorted with patients who have the same active infection or are colonized with the same pathogen.

Healthcare workers involved in the care of patients on contact precautions must wear non-sterile gloves for all patients contact. Gowns are required if there is likely to be substantial direct contact with the patient or any infective material. Gowns and gloves should be removed prior to exiting isolation rooms, and hands must then be washed immediately after patient contact. Medical equipment must be dedicated to a single patient when possible, to avoid transfer of pathogens via fomites. Equipment that must be shared should be cleaned and disinfected for another patient (Esteban et al, 1999).

b- Droplet precautions: droplets are particles of respiratory secretions larger than $5 \mathrm{mi}$ crometers. Because droplets cannot remain suspended in the air for extended periods, exposure of less than three feet (one meter) is usually required for human-to-human transmission of droplet-borne pathogens. However, investigations during the 2003 outbreak of severe acute respiratory syndrome (SARS) suggested that droplets can be transmitted up to six feet from the source patients.

Droplet precautions should be used in the care of patients with suspected or confirmed Neisseria meningitidis, Bordetella pertussis, influenza, adenovirus, Haemophilus influenzae type, Mycoplasma pneumoniae, rubella, and other pathogens spread by droplets. Some organisms (e.g., some respiratory viruses) can be transmitted by both droplets and contact. In this setting, both droplet and contact precautions should be implemented. Healthcare workers within six to ten feet of patients on droplet precautions should wear a facemask. Special air handling systems and use of higher level respirator masks are not required for the care of patients with diseases that are capable of droplet transmission. The doors of rooms used to house the patients must remain open in contrast to the patients on the airborne precautions (CDC, 1994).

c- Airborne precautions: airborne droplet nuclei, in contrast to larger droplets in the preceding section, are particles of respiretory secretions smaller than 5 micrometers that can remain suspended in the air for extended periods and thus can be a source of human inhalational exposure for individuals who are susceptible. Airborne precautions should be used in the care of patients with suspected or confirmed tuberculosis (TB), measles, varicella, small-pox and SARS. TB is principally transmitted in hospitals by droplet nuclei, and the hospital can be an epicenter for the spread of TB to both patients and staff (McDonald et al, 1999). Health care workers immune to measles or varicella need not wear respiratory protection. Airborne precautions are not needed in the setting of herpes zoster infection (Do et al, 1999). Although SARS is transmitted predominantly by droplet spread and direct contact, airborne transmission can occur. The CDC recommends contact and airborne precautions (Cookson and Jarvis, 1997).

Patients on airborne isolation precautions should be placed in an airborne infection isolation room (AIIR). This should be a private room with negative air pressure and a minimum of six to twelve air changes per hour. The door to the isolation rooms must remain closed, and all persons entering must wear a respirator with a filtering capacity of 95\% that allows a tight seal over the nose and mouth. If patients in respiratory isolation require transport outside their isolation rooms for medical procedures, they should wear surgical masks that cover their mouths and noses during transport. The procedures for these patients should be scheduled at times when they can be done rapidly and when waiting areas are less crowded.

d- Immunocompromised patients: Special protective environments are used for pat-i ents undergoing hematopoietic cell transplantation to minimize their exposure to invasive fungal infections such as aspergillo- 
sis. Such protective environments include HEPA filtration of incoming air, positive room air pressure relative to corridors, directed room air flow, ventilation systems that provide at least 12 air changes per hour, dust control measures and the prohibition of flowers and potted plants in patient rooms.

3- Environmental cleaning: Environmental cleaning, disinfection, and sterilization are basic and important measures used to prevent or reduce infections in the hospital environment. The oversight and monitoring of cleaning, sterilization and disinfection practices are direct responsibilities of an infection control unit. The exact definition of each term is important since protocols for each procedure are different and their use in hospital infection control has to be precisely determined (Wilder-Smith and Low, 2005).

a- Cleaning: is the removal of all foreign materials (soil, organic material) from objects, done with water, mechanical action, and detergents or enzymatic products. Meticulous physical cleaning must always precede disinfection and sterilization procedures, and is adequate alone for cleaning items such as blood pressure cuffs (Rutala, 1996). Enforcement of the routine environmental cleaning measures was remarkably effective in reducing vancomycin-resistant enterococcus (VRE) acquisition among the patients in a medical intensive care unit (Hayden et al, 2006).

The importance of environmental decontamination of rooms previously occupied by patients with MRSA and/or VRE in ICUs is illustrated by a retrospective cohort study of patients admitted to eight ICUs. Patients admitted to rooms previously occupied by patients colonized or infected with MRSA or VRE were significantly more likely to become MRSA or VRE colonized than were patients admitted to the rooms whose prior occupant was the MRSA or VRE negative, adjusted odds ratio (AOR) 1.4 for either MRSA or VRE (Huang et al, 2006).

b- Disinfection: describes a process that eliminates many or all pathogenic micro- organisms from inanimate objects, except for bacterial spores. In healthcare settings, this is usually accomplished by the use of liquid chemicals. Efficacy of disinfection is affected by factors as: previous physical cleaning of the object, organic load on the object type and level of microbial contamination, concentration of and exposure time to the germicide and physical configuration of the object Temperature and $\mathrm{pH}$ of the disinfection process

c- Sterilization: is the complete elimination or destruction of all forms of microbial life and is accomplished by either physical or chemical processes. Steam under pressure, dry heat, low temperature sterilization processes (ethylene oxide gas, plasma sterilization), and liquid chemicals are main sterilizing agents used (Collignon et al, 1996). Steam sterilization remains the most widely used technique. A rational approach to disinfection and sterilization of patient care items or equipment was devised in 1968 and still widely used. Patient care items were divided into critical, semi-critical and non-critical categories according to degree of risk of infection involved in their use.

d- Reuse of single-use medical devices: This issue of reuse of the devices labeled "single-use only" has sparked significant debate. These devices generally are costly but reuse might expose patients to crossinfection. But, reuse does occur frequently in order to save costs and minimize waste. An institution or practitioner reusing a disposable device should be able to do the following: The device can be adequately cleaned and sterilized the cleaning process does not adversely affect the quality of the device the device remains safe and effective for its intended use (Rutala and Weber, 2004). These "single-use" devices include: diathermy pencils used in the surgery scissors and forceps used in laparoscopic surgery, sclerosing needles, snares and cytology brushes used in endoscopy cannulas, stoneremoving baskets and balloon dilators used in ERCP, angiography catheters used in both 
cardiology and radiologic imaging, cardiac catheters, pacing electrodes, pacemakers used in cardiology, bone-marrow trephine sets, plasma membrane filters used in hemodialysis an stopcocks used in bronchoscopy.

Satisfactory cleaning and sterilization of a device should theoretically prevent infection transmission and make a device safe to reuse. However, the physical characteristics of the device (especially those constructed with heat-sensitive materials) may not with-stand this process. Any devices with hollow lumens or crevices, or those that cannot be disassembled for cleaning, are very difficult to clean reliably and sterilize; so better by autoclaving or with ethylene oxide. Even when requirements for satisfactory cleaning followed organic material frequently remains on these devices (Wilson et al, 2000).

d- Disease transmission: Iatrogenic transmission of Creutzfeldt-Jakob disease (CJD) occurred in neurosurgical patients when contaminated neurosurgical instruments, such as an implantable electrode that had previously been used in a patient with known CJD, were reused. The electrode had been "sterilized" with $70 \%$ ethanol and formaldehyde vapor, yet two years later electrode was retrieved and implanted into a chimpanzee in which disease subsequently developed. Contaminated neurosurgical instruments were suspected as transmission modes to other patients (Bernoulli et al, 1997). Such agents cannot inactivate CJD prion protein: alcohol, ethylene oxide, formaldehyde, glutaraldehyde, hydrogen peroxide, iodine, ionizing radiation, phenolic, quaternary ammonium compounds, steam sterilization at $121^{\circ} \mathrm{C}$, and urea at concentrations of 6 to $8 \mathrm{~mol} / \mathrm{l}$ (Masters et al, 1979).

e- Nosocomial insect bites: Arthropod bites cause local inflammatory reactions that subside within a few hours. More severe local symptoms, transmission of a disease pathogen, and systemic allergic reactions are also possible. Mosquito bites (Skeeter Syndrome) can cause various local swellings, popular urticarial particularly in children, and rare systemic allergic reactions, including anaphylaxis (Abdel-Motagaly et al, 2017). Popular urticarial is a hyper-sensitivity reaction commonly seen in the children after arthropod bites, although a variety of other bites have been implicated in the smaller numbers of reports. Systemic allergic reactions can occur in response to bites of mosquitoes, several types of blood-sucking flies, fleas, kissing bugs, lice, and ticks (Morsy, 2012). The charge or administrative nurse must examine patient's infestation to find out the hospital's policy as to bedbugs, lice, fleas and scabies. Autoinfection and/or contagious must be considered (Morsy et al, 2000).

Also, the house dust mites (HDM) can be found worldwide as guest in human habitations independent from climate and are a major source of multiple allergens. There was convincing evidence that avoidance of mite allergen could effectively reduce symptoms (Saleh et al, 2013). HDM survive well in mattresses, carpets, furniture and bedding, with figures around 100-500 mites/g dust. Even in dry climates, HDM survive and reproduce easily in bedding (especially in pillows) that takes up moisture from body contact (El-Zemity et al, 2006). HDM allergens are among the commonest triggers of asthma (Adgate et al, 2013). There are at least 15 mite allergens subdivided into groups. G1 \& G2 allergens are the most problematic. G1 consists of proteins with a catalytic activity (e.g., Dermatophagoides pteronyssinus (G1) allergen is a cysteine protease, as is its American counterpart D. farina. G2 are proteins important for mite. Proteins from others affect only few patients (Park et al, 2013).

Recommendations for sterilization require: Pre-cleaning, soaking in 1-2N sodium hydroxide for an hour, followed by steam sterilization at $121^{\circ} \mathrm{C}$ for 30 minutes. Pre-cleaning and sterilization at $132^{\circ} \mathrm{C}$ for 30 minutes; without soaking in sodium hydroxide (Fishman et al, 1998). Instruments used in neuro-surgery on known or suspected cases CJD should not be autoclaved and reused, but should be discarded (Macedo et al, 2005). 
Infection pseudo-outbreaks of from reuse of single-use devices and cross-infection with serious blood-borne pathogens were reported. An independent health research firm concluded that there is no evidence that reuse of single-use devices is either safe or unsafe for patients (Heeg et al, 2001).

The FDA recommended that single-use devices must be reprocessed with the same quality assurance and safety criteria used by the original manufacturer. A group studied whether the reprocessed single-use devices would meet regulatory standards for sterility. Shockingly, they found that none of the reprocessed single-use instruments were effectively cleaned, disinfected, or sterilized. Given the stringency of FDA directives, and the above data, it is likely that hospitals cannot comply with regulations on reuse (Kirkland et al, 1999).

4- Surveillance: Surveillance for nosocomial infections is the cornerstone of all successful hospital infection control programs. The collection of accurate and relevant surveillance data in real time allows infection control practitioners to identify and understand important nosocomial or non-nosocomial (e.g., long-term care facilities) infections or pathogens and to detect epidemics or outbreaks. Detection of clusters of infection often leads to the identification of preexisting systematic problems within the institution, such as surgical practices associated with an increase in surgical site infections (SSI) or failure to change tubing or other equipment on an appropriate schedule.

Surveillance is only the starting point and benchmark for assessing the need for intervention strategies. Surveillance without clear plans to use the data that is collected for the specific and designated purposed is generally unwise. Surveillance can also result in recognition of problems with products or materials with consequences far beyond a single institution. As an example, three early deaths from apparent septic shock in previously healthy individuals undergoing total knee replacement (two) or cartilage graft repla- cement, (one) were reported to the Minnesota Department of Health. A patient had $\mathrm{Cl}$ ostridium sordellii isolated from the blood. Subsequent culture of unplanted tissue from the same source as osteochondral graft also grew Clostridium (Kaye et al, 2006).

A subsequent investigation by personnel from the CDC identified 26 other allograft recipients with clostridial infection. As a result of this investigation, the CDC issued an advisory to tissue processors outlining additional steps to enhance tissue transplant safety and alerted clinicians to consider clostridial sepsis in allograft recipients with evidence of infection following surgery (Richards et al, 2001). Effective surveillance involves counting cases and then calculating rates of various infections, analyzing these data, and then reporting the data in an appropriate way to personnel involved in patient care. The SENIC project of the 1970s demonstrated the importance of per-forming surveillance and developing sys-tems for reporting SSI rates obtained via surveillance back to the hospital's practicing surgeons (Stevenson et al, 2005). They and others concluded that infection control pro-grams that include SSI surveillance coupled with feedback to surgeons, are not only stro-ng and balanced, but are also effective in reducing the SSI rate. Effective-ness of this approach was repeatedly report-ed (Engelhart et al, 2005). Although surveillance is predominantly a component of acute care hospital infection control programs, it is also important in long-term care facilities. Authors have demonstrated infection rates of 3.6 \& 6.0 infections/1000 resident-days respectively in a LTCF in Utah, and a German Nursing Home (Sax and Pittet, 2002). The respiratory tract infections, gastroenteritis, skin and soft tissue infections, and urinary tract infections represented the majority of nosocomial infections. The surveillance in the LTCF, although more challenging than surveillance in the acute cares hospitals, can allow the meaningful comparisons between the facilities (Sax and Pittet, 2002). 
Nosocomial infection rates can also be used to perform comparisons among hospitals. A prevalence study performed over a one-week period in 18 acute care hospitals in Switzerland highlighted a problem with this approach. The unadjusted nosocomial infection rates showed a significantly lower prevalence rate in smaller compared to the intermediate or large hospitals (6.1 vs. 10 \& $10.9 \%$, respectively). But, when case mix was considered in a multivariate analysis, hospital size was no longer a significant factor (Anderson et al, 2007). It is important to note that comparisons among hospitals are only valid when there is adjustment for the overall health of patient and specific surgical factors, as in CDC's National Noso-comial Infection Surveillance System. For example, rates of surgical site infections for many common surgical procedures in small community hospitals (median bed size 220) were different (many were lower; although a few were higher) from rates for same procedures reported (Horan and Emori, 1997).

a- Targeted hospital surveillance: CDC (1972) first proposed hospital-wide surveillance. However, such surveillance was the extremely labor intensive and actually provided few practical data about preventable infections. In 1986, the CDC introduced and promoted the concept of targeted hospital surveillance, and since then, targeted the surveillance has become an essential part of most hospital infection control programs. Targeted surveillance must be defined in each hospital. Ideally, such surveillance should include specific patient populations or units at high risk for infection and/or selected infections that represent the highest priority, most serious consequences, or are highly preventable. The surveillance of rotavirus infections may be a priority in pediatric hospitals, while surveillance of urinary catheter-associated infections may be a focus of targeted surveillance in a hospital that includes a large rehabilitation unit.

b- Components of surveillance: Effective surveillance systems require clear and uni- form definitions of nosocomial infect-ions, such as those developed by the CDC. Uniform definitions allow for comparisons of infection rates both the locally and internationally. Surveillance should also be active and include routine review of the microbiologic, pathologic, clinical and the nursing records. The effective surveillance systems usually include most of the follow-ing components: routine review of microbio-logic records daily, weekly or biweekly rounds on wards, review of selected discharge diagnoses, codes or key words, review of patients who are readmitted following surgical procedures or of patients who undergo reoperation, review of autopsy, radiologic and pathologic reports from nursing, medical or surgical staff members of known or suspected infections. Effective surveillance systems have some degree of redundancy in order to reduce systematic errors (information bias). This redundancy is effective if most or many infections are reported by more than one method. The surveillance of the SSI should rely upon microbiologic data, regular ward rounds by infection control practitioners, and solicitation of reports of known or possible infection from surgeons and nurses during and after hospital discharge (Garner et al, 1988).

c- Surveillance cultures: Another potential component of surveillance is active surveillance cultures. This has primarily been studied for the control of methicillin-resistant St. aureus and vancomycin-resistance enterococci. The recommendations of these guidelines should be followed when caring for patients with MRSA: Place patient in a private room, or place two or more patients with MRSA in same room, wear clean non-sterile gloves when entering patient's room, remove gloves when leaving patient's room, wash hands with an antimicrobial soap or waterless antiseptic agent upon leaving the patient's room, wear a gown when entering the room if substantial contact with the patient or environmental surfaces in the room is anticipated, or if the patient has wound drain- 
age not contained by a dressing, remove the gown before leaving the patient's room, wearing a mask when caring for MRSA patients may reduce the nasal acquisition of MRSA by health care workers and also, upon removing gloves and gown there should be clean hands with an alcohol-based hand rub. But, if hands are visibly contaminated with blood or other proteinaeous materials, wash hands with an antimicrobial soap and water, limit transport of the patient from the room to essential purposes only. When possible, dedicate the use of non-critical equipment to a patient or cohort of patients. If use for another patient is un-avoidable, adequately clean and disinfect the item before use. Implement a program of active surveillance cultures to identify patients colonized or infected with MRSA. Early detection of colonized patients facilitates more timely institution of the appropriate contact precautions (use of gloves \& gowns) that proved to control the MRSA spread more effectively than the standard precautions. Surveillance cultures of anterior nares and open wounds are must for MRSA risky patients of colonization and/or infection.

d- Regulatory bodies: JCAHO is a nongovernmental organization that devises the standards of quality used to accredit hospitals. These standards are varied and range from the responsibilities of the administration to employee education, safety in the workplace, and ethics. The JCAHO mandated in 1970 that all hospitals have infection control committees as a requirement for accreditation. Accreditation is voluntary, although most hospitals view it as mandatory (Taguchi et al, 1991). Occupational Safety and Health Ad-ministration (OSHA), established in 1970, is part of the United States Department of Labor and operate in all states under federal jurisdiction. The OSHA has enforcement activities in the area of preventing occupational risk of blood-borne pathogens, and released the final standard on these exposures, which came into effect in 1992. The standard needs employers to pro- vide hepatitis $\mathrm{B}$ vaccination, personal protective clothing and equipment, post-exposure medical evaluation and follow-up and specific education and training. OSHA conducts periodic inspections of healthcare facilities to check their compliance with several standards and other inspections in response to various hazards and employee complaints (Wenzel, 2002). Quality assessment, risk management and continuous quality improvement. Components of quality monitoring include data collection and analysis, interpretation, remedial action to correct poor quality, and verification that these actions actually improved quality. Quality assessment and risk-management pro-grams were developed primarily by administrators in response to economic forces, particularly the rising costs of care and mal-practice suits. Traditional quality improvement and risk-management programs rely upon empiric case analysis and occurrence of sentinel events, rather than the rates of the specific events in populations. However, this approach has some serious deficiencies. The most serious flaw is the predetermined base-line rates absence, which impairs analysis of the significance of an event and the effect of the intervention. This approach was also not cost effective. Infection control practitioners should be involved in shaping quality assessment movement based upon a solid epidemiologic foundation (Mandell, 2000).

Studying the relationship between a practice and an outcome, as timing of antibiotic administration and rates of SSI, is CQI example of applied to healthcare. Epidemiology should be the cornerstone of CQI that requires a system of careful surveillance, analysis, feedback and education to be the effective (McDonald and Pugliese, 1993).

e- Lives campaign: The Institute of Medicine has initiated a protected 5 million lives campaign that includes measures to reduce certain nosocomial infections. Plan included 5 components: active surveillance cultures, hand hygiene, environmental de-contamination and equipment, contact precautions for 
infected and colonized patients, and the use of device bundles (Herwaldt, 2007).

f- Public reporting: Public reporting of nosocomial infection rates in hospitals is the government-based initiative to reduce infection rates and improving patient safety. The thirty-nine states have introduced legislation and six states have passed the laws requiring disclosure of hospital-acquired infections to the state and, in most cases, to the public. Such public reporting is focused on process and outcome measures (Weinstein et $a l, 2005)$. Reporting of infection rates, CDC focuses on three commonest infections, associated with high morbidity and mortality to be controlled as infections associated with central venous catheters, surgical site infections, and ventilator-associated pneumonia (McKibben et al, 2005).

Nurse's Role: Nurses play a pivotal role in preventing hospital-acquired infections (HAI), not only by ensuring that all aspects of their nursing practice is evidence based, but also through nursing research and patient education.

As patient advocates, nurses are in the unique position to affect change to improve patient care standards. The nurse has many tools available to create a safe environment for patients. Universal precautions are the cornerstones of a safe environment that is free of infection. According to the CDC, (2010) universal precautions are designed to prevent blood borne pathogens transmission when providing first aid or healthcare. They apply to a wide variety of body fluids as blood, cerebrospinal fluid, amniotic fluids, semen and vaginal secretions. They do not apply to the nasal secretions, sputum, saliva, sweat, tears, urine, feces or vomit unless these fluids contain visible blood. Under the universal precautions rule, nurses must wear personal protective equipment when coming into contact with the specified body fluids.

Hand washing is another potent weapon in the nurse's arsenal against infection, and is a single most important nursing intervention to prevent infection. Effective hand washing may be accomplished with antimicrobial soap and water, and specific guidelines are provided by the CDC for the use of alcoholbased hand rubs as acceptable substitutes.

There are many other ways in which nurses can prevent bedside infection. Urinary catheterization avoidance is a must whenever possible. If it is not clinically feasible to avoid catheterization, intermittent catheterized- ion is another preferable option. For patients who require long-term catheterization, suprapubic catheters should be considered. Scrupulous hand washing and aseptic technique is vitally important in the insertion and care of urinary catheters, as well as accurate and precise documentation.

Irrigating cutaneous wounds thoroughly between dressing changes, debriding necrotic material effectively and dressing a wound appropriately to absorb exudates, are all ways in which nurses can protect patients from HAIs.

Neutropenia fever patients must receive frequent oral care, including teeth brushing and gentle flossing, or receive oral antimicrobial rinses when gingivitis or poor hygiene is noted.

Intravenous therapy is a huge area of concern with HAIs. Nurses can make a huge contribution in this war against infection by using full barrier precautions (sterile field, caps, gowns, masks and gloves) when preparing for the insertion of central venous catheters. All catheters, regardless of site, must be placed aseptically. A $2 \%$ chlorhexidine preparation is the preferred cleansing agent of catheter sites and injection ports and diaphragms of multi-dose vials should be cleansed with $70 \%$ ethanol prior to accessing (CDC, 2010). Catheters must be removed when deemed unnecessary.

Catheter dressings should be replaced immediately when damp, soiled or loosened. IV administration sets, extensions and secondary sets must be replaced every $72 \mathrm{hrs}$, unless infection is suspected or documented. Also, to practical bedside interventions, she can foster a safe environment for patients by 
creats an open, non-punitive environment where errors and near misses can be reported. This helps how to improve system and prevent future errors from occurring. Become familiar with organization's error reporting policies, procedures and keep in mind: a- Adopt a safety-minded attitude. Safety is everybody's job! Make prevention a part of your work habits, b- Focus on the task at hand, c- When noise in your environment is distracting, you and others are at risk for accidents d- Noise might include your own thoughts that are unrelated to the task at hand, an interesting conversation going on nearby, or anything that breaks your concentration, e- Identify noise and take actions to limit the source, and f- Develop a personal list-making or note-taking system to keep your thoughts focused.

\section{Recommendations}

Infection control aims to prevent pathogens being passed from one person to another. Good infection control foundation is to assume that everyone is potentially infectious. Basic infection control procedures include hand-washing and keep workplace clean. Research is needed to identify meaningful measures to report best way to record infections, and assess whether such reporting improves patient safety.

Unfortunately, financial restraints, mainly as a larger proportion of population use such facilities, may retard this progress, since basic engineering needs for sinks and single rooms, and increased educational requirements and skills for staff will add to the costs.

\section{References}

Abdel-Motagaly, AME, Mohamad, HM, Morsy, TA, 2017: A mini-review on skeeter syndrome or large local allergy to mosquito bites. J. Egypt. Soc. Parasitol. 47, 2:415-24.

Adgate, JL, Banerjee, S, Wang, M, McKenzie, L, et al, 2013: Performance of dust allergen carpet samplers in controlled laboratory studies. J. Exp. Sci. Environ. Epidemiol. 23, 4:385-91.

Alrawi, S, Houshan, L, Satheesan, R, et al, 2001: Glove reinforcement: an alternative to double gloving. Infect. Cont. Hosp. Epidemiol. 22: 526-34.
Anderson, DJ, Sexton, DJ, Kanafani, ZA, et al, 2007: Severe surgical site infection in community hospitals: Epidemiology, key procedures, and the changing prevalence of methicillin-resistant Staphylococcus aureus. Infect. Cont. Hosp. Epidemiol. 28:1047-54.

Bernoulli, C, Siegfried, J, Baumgartner, G, et $a l$, 1977: Danger of accidental person-to-person transmission of Creutzfeldt-Jakob disease by surgery. Lancet 1:478-86.

Boyce, JM, Pittet, D, 2002: Guideline for Hand Hygiene in Health-Care Settings. Recommendations of the Healthcare Infection Control Practices Advisory Committee and the HICPAC/ SHEA/APIC/IDSA Hand Hygiene Task Force: Society for Healthcare Epidemiology of American Association for Professionals in the Infection Control/ Infectious Diseases Society of America. MMWR. Recom. Rept. 51:1-40.

Bradley, SE, 2005: Double, double, toil and trouble: Infections still-spreading in long-term-care facilities. Infect. Cont. Hosp. Epidemiol. 26:22732.

CDC, 1994: Guidelines for preventing the transmission of Mycobacterium tuberculosis in healthcare facilities. MMWR. Recomm. Rep. 43:1.

CDC, 2010: Healthcare associated infection.

Collignon, PJ, Graham, E, Dreimanis, DE, 1996: Reuse in sterile sites of single-use medical devices: how common is this in Australia? Med. J. Aust. 164:533-9.

Cookson, ST, Jarvis, WR, 1997: Prevention of nosocomial transmission of Mycobacterium tuberculosis. Infect. Dis. Clin. North Am. 11:38594.

Do, AN, Limpakarnjarat, K, Uthaivoravit, W, et al, 1999: Increased risk of Mycobacterium tuberculosis infection related to the occupational exposures of health care workers in Chiang Rai, Thailand. Int. J. Tuberc. Lung Dis. 3:377-84.

Doebbeling, BN, Pfaller, MA, Houston, AK, Wenzel, RP, 1988: Removal of nosocomial pathogens from the contaminated glove: Implications for glove reuse and hand-washing. Ann. Int. Med. 109:394.

Eckmanns, T, Bessert, J, Behnke, M, et al, 2006: Compliance with antiseptic hand rub use in intensive care units: the Hawthorne effect. Infect. Cont. Hosp. Epidemiol. 27:931-40.

Edmond, MB, Wenzel, RP, 2005: Organization for Infection Control. In: Principles and Practice of Infectious Diseases, $6^{\text {th }}$ ed. Mandell, GL, Ben- 
nett, JE, Dolin, R, (eds.), Churchill Livingstone, Philadelphia, PA.

El-Zemity, S, Rezk, H, Farok, S, Zaitoon, A, 2006: Acaricidal activities of some essential oils and their monoterpenoidal constituents against house dust mite, Dermatophagoides pteronyssinus (Acari: Pyroglyphidae). J. Zhejiang Univ. Sci. B7, 12:957-62

Engelhart, ST, Hanses-Derendorf, L, Exner, M, et al, 2005: Prospective surveillance for healthcare-associated infections in German nursing home residents. J. Hosp. Infect. 60:46-9.

Esteban, J, Gadea, I, Roblas, R, et al, 1999: Pseudo-outbreak of Aeromonas hydrophila isolates related to endoscopy. J. Hosp. Infect. 41: 313-7.

Fishman, M, Fort, G, Mikolich, D, 1998: Prevention of Creutzfeldt-Jakob disease in healthcare workers: a case study. Am. J. Infect. Cont. 26:74-8.

Garner, JS, 1996a: Guideline for isolation precautions in hospitals: Evolution of isolation practices, Hospital Infection Control Practices Advisory Committee. Am. J. Infect. Cont. 24:24-8.

Garner, JS, 1996b: Guidelines for isolation precautions, hospital infection control practices advisory committee. Infect. Cont. Hosp. Epidemiol. 17:53-9.

Garner, JS, Jarvis, WR, Emori, TG, et al, 1988: CDC definitions for nosocomial infections, 1988. Am. J. Infect. Cont. 16:128-32.

Gordin, FM, Schultz, ME, Huber, RA, Gill, J A, 2005: Reduction in nosocomial transmission of drug-resistant bacteria after introduction of an alcohol-based handrub. Infect. Cont. Hosp. Epidemiol. 26:650-60.

Gould, DJ, Chudleigh, JH, Moralejo, D, Drey, N, 2007: Interventions to improve hand hygiene compliance in patient care. Cochrane Database Syst. Rev: CD005186.

Haley, RW, Culver, DH, White, JW, et al, 1985: The efficacy of infection surveillance and control programs in preventing nosocomial infection in US Hospitals. Am. J. Epidemiol. 121: 182-8.

Hall, CB, 2007: The spread of influenza and other respiratory viruses: complexities and conjectures. Clin. Infect. Dis. 45:353.

Hayden, MK, Bonten, M, Blom, D, et al, 2006: Reduction in acquisition of vancomycin-resistant enterococcus after enforcement of routine environmental cleaning measures. Clin. Infect. Dis. 42:1552-8.
Heeg, P, Roth, K, Reichl, R, et al, 2001: Decontaminated single-use devices: An oxymoron that may be placing patients at risk for cross-contamination. Infect. Cont. Hosp. Epidemiol 22: 542-6.

Herwaldt, LA, 2007: National issues and future concerns. In: Wenzel, RP, (Ed), Prevention and control of nosocomial infections, $2^{\text {nd }}$ ed. Williams and Wilkins, Baltimore. Institute for the Healthcare Improvement, USA.

Horan, TC, Emori, TG, 1997: Definitions of key terms used in the NNIS System. Am. J. Infect. Cont. 25:112-8.

Huang, SS, Datta, R, Platt, R, 2006: Risk of acquiring antibiotic-resistant bacteria from prior room occupants. Arch. Int. Med. 166:1945.

Kaye, KS, Engemann, JJ, Fulmer, EM, et al, 2006: Favorable impact of an infection control network on nosocomial infection rates in community hospitals. Infect. Cont. Hosp. Epidemiol. 27:228-32.

Kirkland, KB, Briggs, JP, Trivette, SL, et al, 1999: The impact of surgical-site infections in the 1990s: Attributable mortality, excess length of hospitalization, and extra costs. Infect. Cont. Hosp. Epidemiol. 20:72-5.

Larson, E, 1999: Skin hygiene and infection prevention: more of the same or different approaches? Clin. Infect. Dis. 29:1287-90.

Macedo, de, Oliveira, A, White, KL, Leschinsky, DP, et al, 2005: An outbreak of HCV infections among outpatients at a hematology/oncology clinic. Ann. Int. Med. 142:898.

Mandell, D, 2000: Bennett's Principles and Practice of Infectious Diseases. $5^{\text {th }}$ ed. Philadelphia: Churchill Livingstone.

Masters, CL, Harris, JO, Gaidusek, DS, Gibbs, CJ Jr, et al, 1979: Creutzfeldt-Jakob disease: Patterns of worldwide occurrence and the significance of familial and sporadic clustering. Ann. Neurol. 5:177-82.

McDonald, LL, Pugliese, G, 1993: Regulatory, accreditation, and professional agencies influencing infection control programs. In: Wenzel, RP, (ed.), Prevention and control of nosocomial infections, $2^{\text {nd }}$ ed. Williams \& Wilkins, USA.

McDonald, LC, Archibald, LK, et al, 1999: Unrecognized Mycobacterium tuberculosis bacteraemia among hospital inpatients in less developed countries. Lancet 354:115-9.

McKibben, L, Horan, T, Tokars, JI, et al, 2005: Guidance on public reporting of healthcare-associated infections: recommendations of 
the Healthcare Infection Control Practices Advisory Committee. Am. J. Infect. Cont. 33:217-22. Moolenaar, RL, Crutcher, JM, Joaquin, V, et al, 2000: A prolonged outbreak of Pseudomonas aeruginosa in a neonatal intensive care unit: did staff fingernails play a role in disease transmission? Infect. Cont. Hosp. Epidemiol. 21:80-8.

Morsy, TA, 2012: Insect bites and what is eating you? J. Egypt. Soc. Parasitol. 42, 2:291-308.

Morsy, TA, Abou El-Ela, RG, Morsy, AT, Nassar, MI, Khalaf, S, 2000: Two contagious ecto-parasites in orphanage children in Nasr City, Cairo. J. Egypt. Soc. Parasitol. 30, 3:727-34.

Muto, CA, Jernigan, JA, Ostrowsky, BE, et $a l$, 2003: SHEA guideline for preventing nosocomial transmission of multidrug-resistant strains of Staphylococcus aureus and enterococcus. Infect. Cont. Hosp. Epidemiol. 24:362-8.

Noskin, GA, Stosor, V, Cooper, I, Peterson, L R, 1995: Recovery of vancomycin-resistant enterococci on fingertips and environmental surfaces. Infect. Cont. Hosp. Epidemiol 16:577.

Park, SY, Jing, X, Gupta, D, et al, 2013: Peptidoglycan recognition protein 1 enhances experi mental asthma by promoting Th $2 \&$ Th17 and limiting regulatory T cell \& plasmacytoid dendritic cell responses. J. Immunol. 190, 7:3480-92.

Passaro, DJ, Waring, L, Armstrong, R, et al, 1997: Postoperative Serratia marcescens wound infections traced to an out-of-hospital source. J. Infect. Dis. 175:992.

Pittet, D, Hugonnet, S, Harbarth, S, et al, 2000: Effectiveness of a hospital-wide program to improve compliance with hand hygiene: Infection control program. Lancet 356:1307-12.

Pittet, D, Allegranzi, B, Sax, H, et al, 2006: Evidence-based model for hand transmission during patient care and the role of improved practices. Lancet Infect. Dis. 6:641.

Pottinger, J, Burns, S, Manske, C, 1989: Bacterial carriage by artificial versus natural nails. Am. J. Infect. Cont. 17:340.

Rey, JF, 1999: Endoscopic disinfection: a worldwide problem. J. Clin. Gastroenterol. 28:291-9.

Richards, C, Edwards, J, et al, 2001: Feeding back surveillance data to prevent hospital-acquired infections. Emerg. Infect. Dis. 7:295-302.

Rubin, DM, 1988: Prosthetic fingernails in the OR: A research study. AORN J. 47:944-8.

Rupp, ME, Fitzgerald, T, Puumala, S, et al, 2008: Prospective, controlled, cross-over trial of Rupp, ME, Fitzgerald, T, et al, 2008: Prospective, controlled, cross-over trial of alcohol-based hand gel in critical care units. Infect. Cont. Hosp. Epidemiol. 29:8-12.

Rutala, WA, 1996: Guideline for selection and use of disinfectants. Am. J. Infect. Cont. 24:313.

Rutala, WA, Weber, DJ, 2004: Disinfection \& sterilization in health care facilities: what clinicians need to know. Clin. Infect. Dis. 39: 702-8.

Saleh, AMA, Ali, HA, Ahmed, SAM, Mohammad, NM, Morsy, TA, 2013: The effect of an intervention program in nurses' knowledge and performance regarding management of house dust mites in a military hospital. J. Egypt. Soc. Parasitol. 43, 3:821-40

Sax, H, Pittet, D, 2002: Inter-hospital differences in nosocomial infection rates: importance of case-mix adjustment. Arch. Int. Med. 162:24-37.

Siegel, JD, Rhinehart E, Jackson M, et al, 2007: Healthcare Infection Control Practices Advisory Committee 2007 Guideline for isolation precautions: Preventing transmission of infectious agents in healthcare settings (http:// www. cdc.gov/ncidod/dhqp/gl_isolation.html).

Stevenson, KB, Moore, J, Colwell, H, Sleeper, B, 2005: Standardized infection surveillance in long-term care: Inter-facility comparisons from a regional cohort of facilities. Infect. Cont. Hosp. Epidemiol. 26:231-8.

Taguchi, F, Tamai, Y, Uchida, K, et al, 1991: Proposal for a procedure for complete inactivation of the Creutzfeldt-Jakob disease agent. Arch. Virol. 119:297-302.

Taylor, DM, 1996: Creutzfeldt-Jakob disease. Lancet 347:1333.

Weinstein, RA, Siegel, JD, Brennan, PJ, 2005: Infection-control report cards-securing patient safety. N. Engl. J. Med. 353:225-9.

Wenzel, RP, 2002: Organization for infection control. In: Mandell, GL, Bennett, JE, Dolin, R, eds.

Whitehouse, JD, Sexton, DJ, Kirkland, KB, 1998: Infection control: past, present, and future issues. Compr. Ther. 24:71-80.

Wilder-Smith, A, Low, J, 2005: Risk of respiratory infections in healthcare workers: lessons on infection control emerge from the SARS outbreak. Southeast-Asian J. Trop. Med. Pub. Hlth. 36:481-6.

Wilson, SJ, Everts, RJ, Kirkland, et al, 2000: A pseudo-outbreak of Aureobasidium species lower respiratory tract infections caused by reuse of single-use stopcocks during bronchoscopy. Infect. Cont. Hosp. Epidemiol. 21:470-8. 